\begin{tabular}{|r|l|}
\hline \multicolumn{2}{|c|}{ Statistica Sinica Preprint No: SS-2021-0024 } \\
\hline Title & $\begin{array}{l}\text { On Construction of Nonregular Two-Level Factorial } \\
\text { Designs With Maximum Generalized Resolutions }\end{array}$ \\
\hline Manuscript ID & SS-2021-0024 \\
\hline URL & http://www.stat.sinica.edu.tw/statistica/ \\
\hline DOI & $10.5705 /$ ss.202021.0024 \\
\hline Complete List of Authors & $\begin{array}{l}\text { Chenlu Shi and } \\
\text { Boxin Tang }\end{array}$ \\
\hline Corresponding Author & Boxin Tang \\
\hline E-mail & boxint@sfu.ca \\
\hline Notice: Accepted version subject to English editing.
\end{tabular}




\title{
On Construction of Nonregular Two-Level Factorial Designs with Maximum Generalized Resolutions
}

June 22, 2021

\author{
Chenlu Shi \\ chenlu.shi@stat.ucla.edu, UCLA \\ BOXIN TANG \\ boxint@sfu.ca, Simon Fraser University
}

\begin{abstract}
The generalized resolution was introduced and justified in Deng and Tang (1999) as a criterion for selecting nonregular factorial designs. Although there have been extensive research activities on other aspects of nonregular designs, developments on the construction of nonregular designs with maximum generalized resolutions pale by comparison. To date, our knowledge of nonregular designs with maximum generalized resolutions is predominantly computational except very few theoretical results. Devoting the whole paper to this topic, we undertake a comprehensive study on the construction of nonregular designs with maximum generalized resolutions. We derive lower bounds on relevant $J$-characteristics and present construction results. With the assistance of the lower bounds, many of the constructed designs are shown to have maximum generalized resolutions.
\end{abstract}

Key words and phrases: good Hadamard matrix; orthogonal array; Paley construction; tensor product. 


\section{Introduction}

Nonregular factorial designs are, in short, those factorial designs that are not regular and, therefore, cannot be specified by defining relations. The generalized resolution, introduced by Deng and Tang (1999), provides a concise characterization for a two-level nonregular design just as the resolution does for a two-level regular design. As a design selection criterion, the generalized resolution is justifiable from two points of view, one from the properties of projection designs and the other from the biases on the estimation of main effects. Extension of the concept to multi-level designs was examined by Evangelaras, Koukouvinos, Dean and Dingus (2005) and Grömping and Xu (2014).

Studies on nonregular designs started as early as in 1940s when Plackett and Burman (1946) introduced a class of main-effect plans for run sizes that are multiples of four, but did not take off until Lin and Draper (1992), Wang and Wu (1995), Cheng (1995) and Box and Tyssedal (1996) investigated the projection properties of nonregular designs. There have been some extensive research activities since and much of later work on nonregular designs centered around the minimum $G_{2}$-aberration criterion and construction of minimum $G_{2}$-aberration designs - see, for example, Tang and Deng (1999), Xu and Wu (2001), Butler (2003), Xu (2003) and Butler (2004). Parallel flats designs are a class of nonregular designs - see Srivastava and Li (1996), Liao, Iyer and Vecchia (1996) and Mee and Peralta (2000) for some representative papers. Nonregular designs from quaternary codes were considered by, for example, Xu and Wong (2007) and Phoa and Xu (2009). A recent work on constructing strength-three orthogonal arrays is that of Vazquez, Goos 
and Schoen (2019). We refer to Xu, Phoa and Wong (2009) for a general review of topics on nonregular designs.

However, our knowledge of nonregular designs with maximum generalized resolutions is predominantly computational. Some important work along this line includes Deng and Tang (2002), Sun, Li and Ye (2008), Schoen, Eendebak and Nguyen (2010), Bulutoglu and Ryan (2015), and Schoen, Vo-Thanh and Goos (2017). There are very few theoretical results available in the literature. $\mathrm{Xu}$ and Wong (2007) showed that quaternary code designs can be constructed to have a generalized resolution of 3.5. Shi and Tang (2018) obtained a couple of results that allow the construction of designs with large generalized resolutions from good Hadamard matrices.

This paper undertakes a comprehensive and systematic study on the construction of two-level nonregular designs with maximum generalized resolutions. We derive lower bounds on relevant $J$-characteristics and present construction results for designs with large generalized resolutions. With the assistance of the lower bounds, many of the constructed designs are shown to have maximum generalized resolutions. Section 2 introduces notation and background and Section 3 provides some preliminary results on saturated orthogonal arrays. The main results are in Section 4, where we derive a general lower bound on relevant $J$-characteristics and present several constructions of nonregular designs with large generalized resolutions. We show that many of the constructed designs have the maximum generalized resolutions. Section 5 presents another construction of designs with maximum generalized resolutions. Section 6 gives a discussion. 


\section{Notation and Preparation}

A two-level factorial design of $n$ runs for $m$ factors is represented by an $n \times m$ matrix $D=\left(d_{i j}\right)$ with $d_{i j}= \pm 1$, where each column represents a factor and each row specifies a run. Design $D$ is an orthogonal array of strength $t$, denoted by $\mathrm{OA}\left(n, 2^{m}, t\right)$, if the $2^{t}$ level combinations occur with the same frequency in each of its submatrices of $t$ columns. Corresponding to every subset of columns of $D$ is a $J$-characteristic, defined as $J_{u}=$ $\sum_{i=1}^{n} \prod_{j \in u} d_{i j}$ where $u \subseteq\{1,2, \ldots, m\}$. Let $r$ be the smallest positive integer such that $\max _{|u|=r}\left|J_{u}\right|>0$, where $|u|$ is the cardinality of $u$. Then the generalized resolution of design $D$, as defined in Deng and Tang (1999), is

$$
R(D)=r+1-\max _{|u|=r}\left|J_{u}\right| / n
$$

Two-level orthogonal arrays are intimately related to Hadamard matrices. A Hadamard matrix of order $n$ is an $n \times n$ square matrix $H$ with entries \pm 1 such that $H^{T} H=H H^{T}=$ $n I_{n}$, where $I_{n}$ is an identity matrix of order $n$. Therefore, the columns of a Hadamard matrix are mutually orthogonal; so are its rows. The order $n$ of a Hadamard matrix has to be 1, 2 or a multiple of 4. Two Hadamard matrices are isomorphic if one can be obtained from the other by a sequence of isomorphic operations consisting of row permutation, column permutation, sign-switching a row and sign-switching a column. A Hadamard matrix is said to be normalized if its first column only contains entry +1 ; any Hadamard matrix can be normalized by sign-switching those rows that have -1 in the first column. Deleting the first column from a normalized Hadamard matrix of order $n$, we obtain a saturated orthogonal array $\mathrm{OA}\left(n, 2^{n-1}, 2\right)$. 
The simplest construction of Hadamard matrices is by means of tensor product. Let $H=\left(h_{i j}\right)$ and $G$ be Hadamard matrices of orders $n_{1}$ and $n_{2}$, respectively. Then $H \otimes G=$ $\left(h_{i j} G\right)$ is a Hadamard matrix of order $n_{1} n_{2}$. A Hadamard matrix of Sylvester type, which corresponds to a regular design, is simply the $k$-fold tensor product of a Hadamard matrix of order 2 for any integer $k \geq 1$.

Paley (1933) presented two constructions of Hadamard matrices. A Galois field of order $s$ is denoted by $G F(s)=\left\{\alpha_{0}, \alpha_{1}, \ldots, \alpha_{s-1}\right\}$ where $s$ is a prime power. A non-zero element $\alpha$ in $G F(s)$ is called a quadratic residue if $\alpha=\beta^{2}$ for some $\beta$ in $G F(s)$. Now define $\chi(\alpha)=0$ if $\alpha=0, \chi(\alpha)=1$ if $\alpha$ is a quadratic residue, and $\chi(\alpha)=-1$ if $\alpha$ is not a quadratic residue.

Both Paley's constructions use matrix $Q=\left(q_{i j}\right)_{s \times s}$ where $q_{i j}=\chi\left(\alpha_{i}-\alpha_{j}\right)$ for $i, j=$ $0,1, \ldots, s-1$. The first asserts that

$$
H=\left[\begin{array}{cc}
1 & -1_{s}^{T} \\
1_{s} & Q+I_{s}
\end{array}\right]
$$

is a Hadamard matrix of order $s+1$, where $1_{s}$ is a column vector of all ones. The second claims that

$$
H=\left[\begin{array}{cccc}
1 & 1_{s}^{T} & -1 & 1_{s}^{T} \\
1_{s} & Q+I_{s} & 1_{s} & Q-I_{s} \\
-1 & 1_{s}^{T} & -1 & -1_{s}^{T} \\
1_{s} & Q-I_{s} & -1_{s} & -Q-I_{s}
\end{array}\right]
$$

is a Hadamard matrix of order $2(s+1)$ if $s=4 k+1$ for some integer $k$.

We see that, for any given order $n$ that is a multiple of 4, Paley's first construction works if $n-1$ is a prime power while his second construction works if $n / 2-1$ is a prime power that has the form $4 k+1$. 
It should be mentioned that matrix $Q$ is skew-symmetric in Paley's first construction and symmetric in his second construction. The symmetry of $Q$ in Paley's second construction will be needed in Section 5 .

\section{Preliminary results}

Our first set of results concerns saturated orthogonal arrays $\mathrm{OA}\left(n, 2^{n-1}, 2\right)$. The next result provides a lower bound on the value of $\max _{|u|=3}\left|J_{u}\right|$ for such designs.

Proposition 1. Let $D$ be an $O A\left(n, 2^{n-1}, 2\right)$. We then have that

$$
\max _{|u|=3}\left|J_{u}\right| \geq n-8\left\lfloor(n / 8)\left(1-1 /(n-3)^{1 / 2}\right)\right\rfloor,
$$

where $\lfloor x\rfloor$ is the floor function.

Shi and Tang (2018) effectively proved that

$$
\max _{|u|=3,4}\left|J_{u}\right| \geq n-8\left\lfloor(n / 8)\left(1-1 /(n-3)^{1 / 2}\right)\right\rfloor .
$$

Although this result does not imply Proposition 1 directly, a tiny modification to its proof is all we need to prove Proposition 1.

Consider the Hadamard matrix from Paley's first construction given in (1) of Section 2. If we delete the first column, consisting of all ones, we obtain a saturated orthogonal array $\mathrm{OA}\left(n, 2^{n-1}, 2\right)$. This array is called a Paley design in the literature.

Proposition 2. If an $O A\left(n, 2^{n-1}, 2\right)$ is a Paley design, we have that

$$
\max _{|u|=3}\left|J_{u}\right| \leq n-8\left\lceil(1 / 8)\left(n-4-2(n-1)^{1 / 2}\right)\right\rceil,
$$


where $\lceil x\rceil$ is the ceiling function.

For a Paley design, Shi and Tang (2018) effectively established that

$$
\max _{|u|=3,4}\left|J_{u}\right| \leq n-8\left\lceil(1 / 8)\left(n-4-2(n-1)^{1 / 2}\right)\right\rceil .
$$

Proposition 2 is an immediate consequence of this result since $\max _{|u|=3}\left|J_{u}\right| \leq \max _{|u|=3,4}\left|J_{u}\right|$.

If the upper bound in Proposition 2 meets the lower bound in Proposition 1, then Paley design minimizes $\max _{|u|=3}\left|J_{u}\right|$ and thus maximizes the generalized resolution. We can check that this happens for $n=12,20,24,28,32,44,60,72$ and 80 . Therefore, Paley designs for these orders have the maximum generalized resolutions. The values of $\max _{|u|=3 \mid}\left|J_{u}\right|$ for these Paley designs are displayed below:

\begin{tabular}{c|rrrrrrrrr} 
order $n$ & 12 & 20 & 24 & 28 & 32 & 44 & 60 & 72 & 80 \\
\hline $\max _{|u|=3 \mid}\left|J_{u}\right|$ & 4 & 12 & 8 & 12 & 8 & 12 & 12 & 16 & 16.
\end{tabular}

Whether or not Paley designs have the maximum generalized resolutions for other orders remains to be settled. The answer may well be affirmative as we have not found a counterexample. A sharper lower bound on the $\max _{|u|=3}\left|J_{u}\right|$ value would help. Nevertheless, Paley designs provide an attractive class of nonregular designs as they have a generalized resolution bounded below by $3+(8 / n)\left\lceil(1 / 8)\left(n-4-2(n-1)^{1 / 2}\right)\right\rceil$, which converges to 4 as $n$ goes to infinity.

As mentioned right after Proposition 2, in addition to having small $\max _{|u|=3}\left|J_{u}\right|$ values, Paley designs also have small $\max _{|u|=4}\left|J_{u}\right|$ values. This makes Paley designs even more attractive as $\max _{|u|=4}\left|J_{u}\right| / n$ represents the largest correlation of two-factor interactions 
with each other. For example, among all $7570 \mathrm{OA}\left(28,2^{27}, 2\right) \mathrm{s}$ (Schoen, Eendebak and Nguyen 2010), twelve of them have $\max _{|u|=3}\left|J_{u}\right|=12$ and all twelve have the maximum generalized resolution. Only one of these twelve arrays has $\max _{|u|=4}\left|J_{u}\right|=12$ and the other eleven all have $\max _{|u|=4}\left|J_{u}\right|=20$. Not surprisingly, the best one is precisely the Paley design.

\section{Main Results}

We examine the general case in this section and consider an $\mathrm{OA}\left(n, 2^{m}, 2\right)$ for $n / 2 \leq$ $m \leq n-1$. The next result generalizes Proposition 1 .

Theorem 1. Let $D$ be an $O A\left(n, 2^{m}, 2\right)$ where $n / 2 \leq m \leq n-1$. We then have that

$$
\max _{|u|=3}\left|J_{u}\right| \geq L(n, m)=n-8\left\lfloor(n / 8)\left(1-q^{1 / 2}\right)\right\rfloor,
$$

where $q=(2 m-n) /((m-1)(m-2))$.

Proof. Write $D=\left(d_{1}, \ldots, d_{m}\right)$. Then there exist real vectors $e_{1}, \ldots, e_{p}$ with $p=n-m-1$ such that $1_{n} / n^{1 / 2}, d_{1} / n^{1 / 2}, \ldots, d_{m} / n^{1 / 2}, e_{1}, \ldots, e_{p}$ form an orthonormal basis in the $n$ dimensional Euclidean space. Since $d_{1} d_{2}, \ldots, d_{1} d_{m}$ are mutually orthogonal, for any given $k=1, \ldots, p$, we have $\sum_{j=2}^{m}\left|\left\langle d_{1} d_{j} / n^{1 / 2}, e_{k}\right\rangle\right|^{2} \leq 1$, where $\langle x, y\rangle$ denotes the inner product of $x$ and $y$. Noting that $d_{1} d_{1}=1_{n}$ and $\left\langle 1_{n}, e_{k}\right\rangle=0$, we obtain $\sum_{j=1}^{m}\left|\left\langle d_{1} d_{j}, e_{k}\right\rangle\right|^{2} \leq n$. In general, we have $\sum_{j=1}^{m}\left|\left\langle d_{i} d_{j}, e_{k}\right\rangle\right|^{2} \leq n$, for every $i=1, \ldots, m$. Summing over $i$ and removing redundancy gives $\sum_{1 \leq i<j \leq m}\left|\left\langle d_{i} d_{j}, e_{k}\right\rangle\right|^{2} \leq n m / 2$. Summing over $k$ and re-arranging, we 
obtain $\sum_{1 \leq i<j \leq m} \sum_{k=1}^{p}\left|\left\langle d_{i} d_{j}, e_{k}\right\rangle\right|^{2} \leq p n m / 2$. This shows that $\min _{i<j} \sum_{k=1}^{p}\left|\left\langle d_{i} d_{j}, e_{k}\right\rangle\right|^{2} \leq$ $p n /(m-1)$, which allows us to take $i^{*}$ and $j^{*}$ so that $\sum_{k=1}^{p}\left|\left\langle d_{i^{*}} d_{j^{*}}, e_{k}\right\rangle\right|^{2} \leq p n /(m-1)$. Now consider vector $d_{i^{*}} d_{j^{*}}$ under the orthonormal basis $1_{n} / n^{1 / 2}, d_{1} / n^{1 / 2}, \ldots, d_{m} / n^{1 / 2}, e_{1}, \ldots, e_{p}$. We then have that $n=\sum_{j \neq i^{*}, j^{*}}\left|\left\langle d_{i^{*}} d_{j^{*}}, d_{j} / n^{1 / 2}\right\rangle\right|^{2}+\sum_{k=1}^{p}\left|\left\langle d_{i^{*}} d_{j^{*}}, e_{k}\right\rangle\right|^{2}$ due to the orthogonality of $d_{i^{*}} d_{j^{*}}$ to $1_{n}, d_{i^{*}}, d_{j^{*}}$. Using $\left|\left\langle d_{i^{*}} d_{j^{*}}, d_{j}\right\rangle\right| \leq \max _{|u|=3}\left|J_{u}\right|$ and the just established bound on $\sum_{k=1}^{p}\left|\left\langle d_{i^{*}} d_{j^{*}}, e_{k}\right\rangle\right|^{2}$, we obtain that $n^{2} \leq(m-2) \max _{|u|=3}\left|J_{u}\right|^{2}+p n^{2} /(m-1)$. Solving for $\max _{|u|=3}\left|J_{u}\right|$ and using $p=n-1-m$, we obtain that $\max _{|u|=3}\left|J_{u}\right| \geq$ $n q^{1 / 2}$ where $q=(2 m-n) /((m-1)(m-2))$. Theorem 1 then follows by noting that $n-\max _{|u|=3}\left|J_{u}\right|$ is a multiple of 8 .

The lower bound $L(n, m)$ in Theorem 1 reduces to that in Proposition 1 when $m=n-1$ as $q=1 /(n-3)$. For $m=n / 2$, Theorem 1 also gives sensible results as it is easily seen that $L(n, n / 2)=0$ if $n$ is a multiple of 8 and $L(n, n / 2)=4$ otherwise. The case of $m=n / 2$ will be further discussed in Section 5 .

Let $P_{n}$ denote a Paley design of order $n$, which is a saturated design of $n$ runs for $n-1$ factors. Further let $P_{n, m}$ be any design that selects $m$ columns from $P_{n}$ and, for convenience, we still call $P_{n, m}$ a Paley design. In Section 3 , we have proved that $P_{n}$ has the maximum generalized resolution for $n=12,20,24,28,32,44,60,72$, and 80 . Using the bound in Theorem 1, we can establish that for each of these orders, design $P_{n, m}$ also has the maximum generalized resolution for a range of $m$ values. For $n=36$, Paley design is unavailable as $n-1=35$ is not a prime power. Using the results of Shi and Tang (2018) on Hadamard matrices of order 36 , an $\mathrm{OA}\left(36,2^{35}, 2\right)$ with $\max _{|u|=3}\left|J_{u}\right|=12$ 
can be obtained. This design and many of its subdesigns can be shown by Theorem 1 to have the maximum generalized resolution. We present in Table 1 the above results for $n=20,24,28,32,36,44,60,72$ and 80 . The case of $n=12$ is excluded from Table 1 because, trivially, $P_{12, m}$ has the maximum generalized resolution for any $m \geq 3$.

Table 1 also includes other designs with the maximum generalized resolutions, obtained later in this paper.

Table 1. Nonregular designs with the maximum generalized resolutions

\begin{tabular}{|c|c|c|c|}
\hline$n$ & $\max _{|u|=3}\left|J_{u}\right|$ & $m$ & source \\
\hline 20 & 12 & 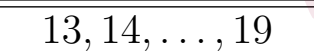 & Theorem 1 \\
\hline 24 & 8 & $13,14, \ldots, 23$ & Theorem 1 \\
\hline 28 & 12 & $17,18, \ldots, 27$ & Theorem 1 \\
\hline 32 & 8 & $17,18, \ldots, 31$ & Theorem 1 \\
\hline 36 & 12 & $21,22, \ldots, 35$ & Theorem 1 \\
\hline 44 & 12 & $25,26, \ldots, 43$ & Theorem 1 \\
\hline 60 & 12 & $33,34, \ldots, 59$ & Theorem 1 \\
\hline 72 & 16 & $52,53, \ldots, 71$ & Theorem 1 \\
\hline 80 & 16 & $54,55, \ldots, 79$ & Theorem 1 \\
\hline 48 & 8 & $25,26, \ldots, 44$ & Theorem 4, Example 3 \\
\hline 64 & 16 & $52,53, \ldots, 62$ & Theorem 3, Example 4 \\
\hline 96 & 16 & $60,61, \ldots, 92$ & Theorem 4 \\
\hline 128 & 16 & $75,76, \ldots, 124$ & Theorem 4, Example 4 \\
\hline 144 & 16 & $83,84, \ldots, 121$ & Theorem 2, Example 1 \\
\hline 192 & 16 & $106,107, \ldots, 176$ & Theorem 4, Example 3 \\
\hline 768 & 32 & $511,512, \ldots, 704$ & Theorem 4, Example 3 \\
\hline 20 & 4 & $3,4, \ldots, 10$ & Theorem 5 \\
\hline 28 & 4 & $3,4, \ldots, 14$ & Theorem 5 \\
\hline 36 & 4 & $3,4, \ldots, 18$ & Theorem 5 \\
\hline 52 & 4 & $3,4, \ldots, 26$ & Theorem 5 \\
\hline 60 & 4 & $3,4, \ldots, 30$ & Theorem 5 \\
\hline 76 & 4 & $3,4, \ldots, 38$ & Theorem 5 \\
\hline 84 & 4 & $3,4, \ldots, 42$ & Theorem 5 \\
\hline 100 & 4 & $3,4, \ldots, 50$ & Theorem 5 \\
\hline 108 & 4 & $3,4, \ldots, 54$ & Theorem 5 \\
\hline 124 & 4 & $3,4, \ldots, 62$ & Theorem 5 \\
\hline
\end{tabular}


The next major task of this section is to present some constructions of nonregular designs with large generalized resolutions. Using Theorem 1, many of the resulting designs are shown to have the maximum generalized resolutions.

Theorem 2. Let $A$ be an $O A\left(n_{1}, 2^{m_{1}}, 2\right)$ with $R(A)=4-\epsilon_{1}$ and $B$ be an $O A\left(n_{2}, 2^{m_{2}}, 2\right)$ with $R(B)=4-\epsilon_{2}$. Then $D=A \otimes B$ is an $O A\left(n_{1} n_{2}, 2^{m_{1} m_{2}}, 2\right)$ with $R(D)=4-\epsilon_{1} \epsilon_{2}$.

Proof. Write $A=\left(a_{1}, a_{2}, \ldots, a_{m_{1}}\right)$ and $B=\left(b_{1}, b_{2}, \ldots, b_{m_{2}}\right)$. Then the columns of $D=$ $A \otimes B$ have the form of $a_{i} \otimes b_{j}$. By a result of Tang (2006), we have

$$
J\left(a_{i_{1}} \otimes b_{j_{1}}, a_{i_{2}} \otimes b_{j_{2}}, a_{i_{3}} \otimes b_{j_{3}}\right)=J\left(a_{i_{1}}, a_{i_{2}}, a_{i_{3}}\right) J\left(b_{j_{1}}, b_{j_{2}}, b_{j_{3}}\right),
$$

where $J(x, y, z)$ is the $J$-characteristic of three column vectors $x=\left(x_{1}, \ldots, x_{n}\right), y=$ $\left(y_{1}, \ldots, y_{n}\right)$ and $z=\left(z_{1}, \ldots, z_{n}\right)$, defined as $J(x, y, z)=\sum_{i=1}^{n} x_{i} y_{i} z_{i}$. It is obvious that $J\left(a_{i_{1}}, a_{i_{2}}, a_{i_{3}}\right)=0$ if $a_{i_{1}}=a_{i_{2}}$ because $A$ is an orthogonal array. We therefore have that $\max _{|u|=3}\left|J_{u}(D)\right|=\max _{|u|=3}\left|J_{u}(A)\right| \max _{|u|=3}\left|J_{u}(B)\right|$. Theorem 2 now follows.

Example 1. Let $A$ and $B$ both be $P_{12}$, an $O A\left(12,2^{11}, 2\right)$, which has a generalized resolution $R=4-1 / 3$. Then Theorem 2 says that design $D=A \otimes B$, an $O A\left(144,2^{121}, 2\right)$ has a generalized resolution $R(D)=4-1 / 9$. In terms of $J$-characteristics, we have that $\max _{|u|=3}\left|J_{u}(D)\right|=16$. As $L(144,121)=16$ by Theorem 1, design $D$ has the maximum generalized resolution. Deleting some columns from $D$ gives a design with less factors. Since $L(144, m)=16$ for all $m$ values in the range of $83 \leq m \leq 121$, we therefore obtain an $O A\left(144,2^{m}, 2\right)$ with the maximum generalized resolution for every $m=83,84, \ldots, 121$. 
Example 2. Let $A$ be $P_{12}$ and $B$ be $P_{32}$, so $R(A)=4-1 / 3$ and $R(B)=4-1 / 4$. Using Theorem 2, we obtain a design $D=A \otimes B$, an $O A\left(384,2^{341}, 2\right)$, which has a generalized resolution $R=4-1 / 12$. This design does not achieve the lower bound in Theorem 1 . Although we do not know if it has the maximum generalized resolution, we do know that it has a large generalized resolution, which is close to 4.

The next result has a flavor similar to that of Theorem 2 but offers a different perspective on the tensor product construction. Given a Hadamard matrix $H$, we define

$$
\gamma(H)=\max _{|u|=1,3}\left|J_{u}(H)\right|
$$

Theorem 3. Let $H$ be a Hadamard matrix of order $n_{1}$ with $\gamma=\gamma(H)$ and $A$ be an $O A\left(n_{2}, 2^{m}, 2\right)$ with $R(A)=4-\epsilon$. Then design $D=H \otimes A$ is an $O A\left(n_{1} n_{2}, 2^{n_{1} m}, 2\right)$ with $R(D)=4-\epsilon\left(\gamma / n_{1}\right)$

Proof. It is obvious that $D=H \otimes A$ is an $\mathrm{OA}\left(n_{1} n_{2}, 2^{n_{1} m}, 2\right)$. To prove $R(D)=4-\epsilon\left(\gamma / n_{1}\right)$, again using Lemma 2 of Tang (2006), we obtain

$$
\max _{|u|=1,3}\left|J_{u}(D)\right|=\max _{|u|=1,3}\left|J_{u}(H)\right| \max _{|u|=1,3}\left|J_{u}(A)\right|
$$

Because $A$ and $D$ are orthogonal arrays of strength two, we have that $J_{u}(D)=J_{u}(A)=0$ for all $u$ with $|u|=1$. This shows that $\max _{|u|=3}\left|J_{u}(D)\right|=\max _{|u|=1,3}\left|J_{u}(H)\right| \max _{|u|=3}\left|J_{u}(A)\right|$, which entails that $R(D)=4-\epsilon\left(\gamma / n_{1}\right)$.

If $H$ in Theorem 3 has a column of all ones, then $\gamma(H)=n_{1}$, and thus $R(D)=4-\epsilon$. This special case was known in Shi and Tang (2018). 
One can easily check that the following Hadamard matrix

$$
H=\left[\begin{array}{rrrr}
-1 & 1 & 1 & 1 \\
1 & -1 & 1 & 1 \\
1 & 1 & -1 & 1 \\
1 & 1 & 1 & -1
\end{array}\right]
$$

has that $\gamma(H)=2$.

Theorem 4. Consider the above Hadamard matrix $H$ of order 4 and let $A$ be an $O A\left(n, 2^{m}, 2\right)$ with $R(A)=4-\epsilon$. Define $D_{0}=A$, and $D_{k}=H \otimes D_{k-1}$ for $k \geq 1$. Then $D_{k}$, an $O A\left(n 4^{k}, 2^{m 4^{k}}, 2\right)$, has that $R\left(D_{k}\right)=4-\epsilon / 2^{k}$ for any integer $k \geq 0$.

Theorem 4 is immediate from Theorem 3.

Example 3. If we take $P_{12}$ as $A$, then $D_{k}$ is an $O A\left(12 \cdot 4^{k}, 2^{11 \cdot 4^{k}}, 2\right)$, which has $R\left(D_{k}\right)=$ $4-(1 / 3) / 2^{k}$ for $k \geq 1$. We also have that $\max _{|u|=3}\left|J_{u}\left(D_{k}\right)\right|=4 \cdot 2^{k}=2^{k+2}$. Now consider $D_{1}, D_{2}$ and $D_{3}$. Design $D_{1}$ is an $O A\left(48,2^{44}, 2\right)$ with $\max _{|u|=3}\left|J_{u}\left(D_{1}\right)\right|=8$. As $L(48, m)=$ 8 for $25 \leq m \leq 44$, any design by selecting $m$ columns from $D_{1}$ has the maximum generalized resolution of $4-1 / 6$. Similarly, we obtain from $D_{2}$ an $O A\left(192,2^{m}, 2\right)$ with the maximum generalized resolution for any $m$ with $106 \leq m \leq 176$, and from $D_{3}$ an $O A\left(768,2^{m}, 2\right)$ with the maximum generalized resolution for any $m$ with $511 \leq m \leq 704$.

Example 4. If we take $P_{32}$ as $A$, then $D_{k}$ is an $O A\left(32 \cdot 4^{k}, 2^{31 \cdot 4^{k}}, 2\right)$, which has that $R\left(D_{k}\right)=4-(1 / 4) / 2^{k}=4-1 / 2^{k+2}$ and that $\max _{|u|=3}\left|J_{u}\left(D_{k}\right)\right|=8 \cdot 2^{k}=2^{k+3}$. Again using the lower bound in Theorem 1, we obtain from $D_{1}$ an $O A\left(128,2^{m}, 2\right)$ that has the maximum generalized resolution for any $m$ with $75 \leq m \leq 124$. 
Now let $C_{k}=\left((1,1)^{T},(1,-1)^{T}\right)^{T} \otimes D_{k}$ for $k \geq 0$. Then $C_{k}$ is $O A\left(64 \cdot 4^{k}, 2^{31 \cdot 2^{2 k+1}}, 2\right)$ also with $R\left(C_{k}\right)=4-1 / 2^{k+2}$ by Theorem 3. Together, designs $D_{k}$ and $C_{k}, k \geq 0$, cover all run sizes $n \geq 32$ that are powers of 2. In this regard, the quaternary code designs, constructed by Xu and Wong (2007) for run sizes that are powers of 2, are not so competitive, since they have a generalized resolution of 3.5.

Application of Theorem 3 requires a Hadamard matrix $H$ with a small value of $\gamma(H)$ defined in (3). A full investigation of this problem is out of the scope of the present paper. Notwithstanding, we provide some results from a preliminary study.

Proposition 3. (i) The value of $\gamma(H)$ must be even.

(ii) If a Hadamard matrix of order $n \geq 4$ exists, we can find one such that $\gamma(H) \leq n-2$.

(iii) We have that $\gamma\left(H_{1} \otimes H_{2}\right)=\gamma\left(H_{1}\right) \gamma\left(H_{2}\right)$ for any two Hadamard matrices $H_{1}$ and $H_{2}$.

Proof. Parts (i) and (iii) are obvious. To prove part (ii), let $H$ be a Hadamard matrix of order $n$ that contains a column of all ones. Then $\gamma(H)=n$. Now we switch the signs of one row of $H$ and obtain another Hadamard matrix, say $H^{\prime}$. Since $J_{u}(H)$ is a multiple of 4 for all $u$ with $|u|=1,3$ and $J_{u}\left(H^{\prime}\right)$ differs from $J_{u}(H)$ by \pm 2 , we have that $J_{u}\left(H^{\prime}\right)$ is even but not a multiple of 4 so $\gamma\left(H^{\prime}\right) \leq n-2$.

Let $\gamma_{n}=\min _{H} \gamma(H)$ where the minimization is over all Hadamard matrices of order $n$. Obviously, we have that $\gamma_{2}=\gamma_{4}=2$. By a computer search, we obtain that $\gamma_{8}=4$ and $\gamma_{12}=8$. A useful lower bound on $\gamma_{n}$ is provided here.

Proposition 4. We have that $\gamma_{n} \geq n^{1 / 2}$. 
Proof. Let $H=\left(h_{1}, \ldots, h_{n}\right)$ be a Hadamard matrix of order $n$. Considering vector $1_{n}$ under the orthonormal basis $\left\{h_{1} / n^{1 / 2}, \ldots, h_{n} / n^{1 / 2}\right\}$, we obtain that $n^{2}=\sum_{j=1}^{n}\left|J\left(h_{j}\right)\right|^{2}$. As $\left|J\left(h_{j}\right)\right| \leq \gamma(H)$, we obtain $\gamma(H) \geq n^{1 / 2}$. Therefore $\gamma_{n} \geq n^{1 / 2}$.

Using the bound in Proposition 4, in conjunction with the fact that $\gamma_{n}$ is even, we have that $\gamma_{16} \geq 4$, and $\gamma_{n} \geq 6$ for $n=20,24,28,32$ and 36 . For $n=4^{k}$, we have that $\gamma_{4^{k}} \geq 2^{k}$. Using the Hadamard matrix of order 4 given in (4) and Part (iii) of Proposition 3, we can construct a Hadamard matrix $H$ of order $4^{k}$ with $\gamma(H)=2^{k}$. This establishes that $\gamma_{4^{k}}=2^{k}$, which means that $D_{k}$ in Theorem 4 is the best if $A$ is also the best.

\section{Further Results}

This section provides another construction of nonregular designs with maximum generalized resolutions. The focus here is on designs with $\max _{|u|=3}\left|J_{u}\right|=4$ when $n$ is not a multiple of 8 .

Consider an $\operatorname{OA}\left(n, 2^{m}, 2\right)$ when $n$ is not a multiple of 8. From Deng and Tang (2002), we know that $J_{u}$ is also not a multiple of 8 for all $u$ with $|u|=3$. This means that $\max _{|u|=3}\left|J_{u}\right| \geq 4$. The best situation is $\max _{|u|=3}\left|J_{u}\right|=4$ which happens if and only if $\left|J_{u}\right|=4$ for all $|u|=3$. An $\operatorname{OA}\left(n, 2^{m}, 2\right)$ with $\left|J_{u}\right|=4$ for all $|u|=3$ has a maximum generalized resolution of $4-4 / n$.

For $n=12$, design $P_{12}$, an $\mathrm{OA}\left(12,2^{11}, 2\right)$, has $\left|J_{u}\right|=4$ for $|u|=3$. For $n=20,28$ and 36, computational results from Deng and Tang (2002), Sun, Li and Ye (2008) and Schoen, Vo-Thanh and Goos (2017) showed that $\operatorname{OA}\left(n, 2^{m}, 2\right) \mathrm{s}$ with $\max _{|u|=3}\left|J_{u}\right|=4$ 
can be found for $m \leq n / 2$. This is no coincidence as the following general result can be established.

Let $n=8 k+4$. Suppose that $s=n / 2-1=4 k+1$ is a prime power. Consider the following two matrices

$$
C=\left[\begin{array}{cc}
-1 & 1_{s}^{T} \\
1_{s} & Q-I_{s} \\
-1 & -1_{s}^{T} \\
-1_{s} & -Q-I_{s}
\end{array}\right], \quad D=\left[\begin{array}{cc}
-1 & 1_{s}^{T} \\
1_{s} & Q-I_{s} \\
1 & 1_{s}^{T} \\
-1_{s} & -Q-I_{s}
\end{array}\right]
$$

where $Q$ is given in Section 2. We see that matrix $C$ is an $n \times(n / 2)$ submatrix of the Hadamard matrix from Paley's second construction, and thus has orthogonal columns. But $C$ is not an orthogonal array because all of its columns have more -1 s than +1 s. Matrix $D$ is an $\operatorname{OA}\left(n, 2^{n / 2}, 2\right)$, obtained from $C$ by multiplying -1 to the $(n / 2+1)$ th row of $C$ while leaving all other rows untouched.

Theorem 5. Suppose that $n / 2-1=4 k+1$ is a prime power. Then the above design $D$ as in (5) is an $O A\left(n, 2^{n / 2}, 2\right)$ with $\max _{|u|=3}\left|J_{u}\right|=4$ and therefore has a maximum generalized resolution $R=4-4 / n$.

Proof. We need to show that $\left|J_{u}\right|=4$ for all $|u|=3$. We note that a projection design of $D$ onto three factors has many mirror image runs. When calculating the $J$ value of a projection design onto three factors, these mirror image runs do not contribute, so we only need to focus on those runs that have no mirror images. Looking at the projection design onto the first three factors, we see that only the following six runs need to be considered: runs $1,2,3$ and runs $(n / 2+1),(n / 2+2)$ and $(n / 2+3)$. Using the symmetry of matrix 
$Q$, it can be easily seen that the $J$ value for the three columns of the matrix consisting of these six runs must be \pm 4 . For other projection designs onto three factors, without loss of generality we consider the projection design onto columns 2, 3 and 4. For this projection design, we see that we only need to consider runs $1,2,3,4$ and runs $(n / 2+1),(n / 2+2)$, $(n / 2+3),(n / 2+4)$. Due to the symmetry of $Q$, matrix $A_{1}$ collecting runs $1,2,3,4$ as rows and matrix $A_{2}$ collecting runs $(n / 2+1),(n / 2+2),(n / 2+3),(n / 2+4)$ as rows must have forms

$$
A_{1}=\left[\begin{array}{rrr}
1 & 1 & 1 \\
-1 & q_{1} & q_{2} \\
q_{1} & -1 & q_{3} \\
q_{2} & q_{3} & -1
\end{array}\right], \quad A_{2}=\left[\begin{array}{rrr}
1 & 1 & 1 \\
-1 & -q_{1} & -q_{2} \\
-q_{1} & -1 & -q_{3} \\
-q_{2} & -q_{3} & -1
\end{array}\right]
$$

The $J$ value for the three columns of the $8 \times 3$ matrix obtained by combining the rows of $A_{1}$ and $A_{2}$ is therefore given by $2-2\left(q_{1} q_{2}+q_{1} q_{3}+q_{2} q_{3}\right)$, which is equal to \pm 4 for all possible scenarios of $q_{1}= \pm 1, q_{2}= \pm 1$ and $q_{3}= \pm 1$. We have therefore shown that $J_{u}= \pm 4$ for all $|u|=3$.

For $n \leq 128$, Theorem 5 allows the construction of an $\mathrm{OA}\left(n, 2^{n / 2}, 2\right)$ with a generalized resolution $R=4-4 / n$ for $n=20,28,36,52,60,76,84,100,108$ and 124 . Obviously, designs from deleting some columns from this $\mathrm{OA}\left(n, 2^{n / 2}, 2\right)$ have the same generalized resolution.

An inescapable question arising from Theorem 5 is how large $m$ can be if an $\operatorname{OA}\left(n, 2^{m}, 2\right)$ is to have $R=4-4 / n$ when $n=8 k+4$. Theorem 5 says that $m \geq n / 2$ if $n / 2-1=4 k+1$ is a prime power. The next result gives an upper bound on $m$. 
Theorem 6. If an $O A\left(n, 2^{m}, 2\right)$ is to have $\max _{|u|=3}\left|J_{u}\right|=4$ for $n=8 k+4 \geq 20$, then it is necessary that $m \leq n / 2+2$.

Proof. We only need to prove that $L(n, m) \geq 12$ for $m=n / 2+3$, where $L(n, m)$ is the lower bound on $\max _{|u|=3}\left|J_{u}\right|$ in Theorem 1 . As $n=8 k+4$, we have that $L(n, m)=$ $(8 k+4)-8\left\lfloor k+0.5-(n / 8) q^{1 / 2}\right\rfloor$. Thus, to prove $L(n, m) \geq 12$, we only need to prove that $0.5-(n / 8) q^{1 / 2}<0$, which is equivalent to $q>(4 / n)^{2}$. Plugging-in $n=8 k+4$ and $m=n / 2+3=4 k+5$ into $q>(4 / n)^{2}$ and then doing some elementary algebra, we obtain an equivalent inequality $2 k(2 k-1)>3$. This last inequality holds true whenever $k \geq 2$ and correspondingly $n=8 k+4 \geq 20$.

A weaker version than Theorem 6 can be proved using results from supersaturated designs. Suppose that there exists a design of $n=8 k+4$ runs and $m$ factors with $\left|J_{u}\right|=4$ for all $|u|=3$. By half fractioning (Lin 1993), we can construct a supersaturated design of $n / 2$ runs for $m-1$ factors with the property that the inner product of any two columns is \pm 2 . By Cheng and Tang (2001, Theorem 4 ), we obtain that $m-1 \leq n / 2+2$ and hence that $m \leq n / 2+3$

Theorem 6 cannot be improved by just using Theorem 1, as we can show in a way similar to proving Theorem 6 that $L(n, n / 2+2)=4$. So, the existence of an $\mathrm{OA}\left(n, 2^{n / 2+1}, 2\right)$ or an $\mathrm{OA}\left(n, 2^{n / 2+2}, 2\right)$ with $R=4-4 / n$ for $n \geq 44$ is still theoretically possible, even though the impossibility has been established for $n=20,28$ and 36 by Sun, Li and Ye (2008) and Schoen, Vo-Thanh and Goos (2017).

When $n$ is a multiple of 8 , an $\operatorname{OA}\left(n, 2^{n / 2}, 3\right)$, an strength-three array, can be con- 
structed by folding over a Hadamard matrix of order $n / 2$. Shi and Tang (2018) showed that if a Hadamard matrix of order $n / 2$ has type $b_{\max }$, then its foldover has the maximum generalized resolution given by $R=4+8 b_{\max } /(n / 2)=4+16 b_{\max } / n$.

\section{Discussion}

This paper provides a comprehensive study on the construction of nonregular designs with maximum generalized resolutions. We derive lower bounds on the value of $\max _{|u|=3}\left|J_{u}\right|$, and present several methods of constructing designs with large generalized resolutions. With the help of lower bounds, many designs are shown to have the maximum generalized resolutions. The following summary provides a simple and clear picture of the previous results in relation with the new results obtained in this paper.

- From the computational results of Sun, Li and Ye (2008), Schoen, Eendebak and Nguyen (2010), and Schoen, Vo-Thanh and Goos (2017), we can deduce that designs with the maximum generalized resolution have been obtained for $n=12,16,20$ and 24 with all $m \leq n-1$, for $n=28$ with $m \leq 14$ and $m=27$, for $n=32$ with $17 \leq m \leq 31$, and for $n=36$ with $m \leq 18$. All the other designs in Table 1 are new.

- The general result of Xu and Wong (2007) is that QC designs of $n$ runs they constructed have a generalized resolution of 3.5, where $n$ is a power of 2 . In contrast, our design $D_{k}$, an $\mathrm{OA}\left(32 \cdot 4^{k}, 2^{31 \cdot 4^{k}}, 2\right)$, given in Example 4 has $R\left(D_{k}\right)=4-1 / 2^{k+2}$; our design $C_{k}$, an $\mathrm{OA}\left(64 \cdot 4^{k}, 2^{31 \cdot 2^{2 k+1}}, 2\right)$, also given in Example 4 has $R\left(C_{k}\right)=4-1 / 2^{k+2}$. 
Together, designs $D_{k}$ and $C_{k}, k \geq 0$, cover all run sizes $n \geq 32$ that are powers of 2 .

One potential future work is to examine the minimum $G_{2}$-aberration properties of the constructed designs, and more specifically to select those designs using the $G_{2}$-aberration criterion from among the constructed designs that have large or maximum generalized resolutions. Hadamard matrices of type $b_{\max }$ can be used to construct saturated strengththree orthogonal arrays with maximum generalized resolutions but the general problem of constructing strength-three arrays with maximum generalized resolutions is yet to be solved. This is an important topic for further investigation.

Acknowledgement. The research of Boxin Tang is supported by the Natural Sciences and Engineering Research Council of Canada.

\section{References}

Box, G.E.P. and Tyssedal, J. (1996). Projective properties of certain orthogonal arrays. Biometrika 83 950-955.

Bulutoglu, D. A. and Cheng, C. S. (2003). Hidden projection properties of some nonregular fractional factorial designs and their applications. Annals of Statistics 31, 1012-1026.

Bulutoglu, D. A. and Ryan, K. J. (2015). Algorithms for finding generalized minimum aberration designs. Journal of Complexity 31, 577-589.

Butler, N. A. (2003). Minimum aberration construction results for nonregular two-level fractional factorial designs. Biometrika 90, 891-898. 
Butler, N. A. (2004). Minimum $G_{2}$-aberration properties of two-level foldover designs. Statistics \& Probability Letters 67, 121-132.

Cheng, C.S. (1995). Some projection properties of orthogonal arrays. Annals of Statistics 23 1223-1233.

Cheng, C. S. and Tang, B. (2001). Upper bounds on the number of columns in supersaturated designs. Biometrika 88, 1169-1174.

Deng, L. Y. and Tang, B. (1999). Generalized resolution and minimum aberration criteria for Plackett-Burman and other nonregular factorial designs. Statistica Sinica 9, 10711082.

Deng, L. Y. and Tang, B. (2002). Design selection and classification for Hadamard matrices using generalized minimum aberration criteria. Technometrics 44, 173-184.

Evangelaras, H., Koukouvinos, C., Dean, A. M., and Dingus, C. A. (2005). Projection properties of certain three level orthogonal arrays. Metrika 62, 241-257.

Grömping, U. and Xu, H. (2014). Generalized resolution for orthogonal arrays. Annals of Statistics 42, 918-939.

Kharaghani, H. and Tayfeh-Rezaie, B. (2013). Hadamard matrices of order 32. Journal of Combinatorial Designs 21, 212-221.

Kimura, H. (1994). Classification of Hadamard matrices of order 28. Discrete Math. 133, 171-180.

Liao, C.T., Iyer, H.K. and Vecchia, D.F. (1996). Construction of orthogonal two-level designs of user-specified resolution where $N \neq 2^{k}$. Technometrics $\mathbf{3 8}, 342-353$. 
Lin, D. K. J. (1993). A new class of supersaturated designs. Technometrics 35, 28-31.

Lin, D.K.J. and Draper, N.R. (1992). Projection properties of Plackett and Burman designs. Technometrics 34 423-428.

Mee, R.W. and Peralta, M. (2000). Semifolding $2^{k-p}$ Designs. Technometrics 42, 122-134.

Paley, R. E. A. C. (1933). On orthogonal matrices. J. Math. Phys. 12, 311-320.

Phoa, F.K. and Xu, H. (2009). Quarter-fraction factorial designs constructed via quaternary codes. The Annals of Statistics, 2561-2581.

Plackett, R.L. and Burman, J.P. (1946). The design of optimum multi-factorial experiments. Biometrika 33 305-325.

Schoen, E.D., Eendebak, P.T. and Nguyen, M.V.M. (2010). Complete enumeration of pure-level and mixed-level orthogonal arrays. Journal of Combinatorial Designs, 18, 123-140.

Schoen, E.D., Vo-Thanh, N. and Goos, P. (2017). Two-level orthogonal screening designs with 24, 28, 32, and 36 runs. Journal of the American Statistical Association 112, 1354-1369.

Shi, C. and Tang, B. (2018). Designs from good Hadamard matrices. Bernoulli 24, 661-671.

Srivastava, J.N. and Li, J. (1996). Orthogonal designs of parallel flats type. Journal of statistical planning and inference 53, 261-283. 
Sun, D.X., Li, W., and Ye, K.Q. (2008). Algorithmic construction of catalogs of nonisomorphic two-level orthogonal designs for economic run sizes. Statistics and Applications, 6, 141-155.

Tang, B. (2006). Orthogonal arrays robust to nonnegligible two-factor interactions. Biometrika 93, 137-146.

Tang, B. and Deng, L. Y. (1999). Minimum $G_{2}$-aberration for nonregular fractional factorial designs. Annals of Statistics 27, 1914-1926.

Vazquez, A.R., Goos, P. and Schoen, E.D. (2019). Constructing two-level designs by concatenation of strength-3 orthogonal arrays. Technometrics 61, 219-232.

Wang, J.C. and Wu, C.F.J. (1995). A hidden projection property of Plackett-Burman and related designs. Statistica Sinica 5 235-250.

Xu, H. (2003). Minimum moment aberration for nonregular designs and supersaturated designs. Statistica Sinica 13, 691-708.

Xu, H., Phoa, F.K. and Wong, W.K. (2009). Recent developments in nonregular fractional factorial designs. Statistics Surveys, 3, 18-46.

Xu, H. and Wong, A. (2007). Two-level nonregular designs from quaternary linear codes. Statistica Sinica 17, 1191-1213.

Xu, H. and Wu, C. J. (2001). Generalized minimum aberration for asymmetrical fractional factorial designs. Annals of Statistics 29, 1066-1077. 